preserved left ventricular function, when compared with the pulsatile device. The cascade of unique physiologic events that we confronted in the management of our patient was a singular learning event that leads to this case report.

\section{References}

1. Carvera R, Font J, Pare C, et al. Cardiac disease in systemic lupus erythematosus: prospective study of 70 patients. Ann Rheum Dis. 1992;51:156-9.

2. Bouma W, Klinkenberg TJ, Van der Horst I, et al. Mitral valve surgery for mitra regurgitation caused by Lieman-Sacks endocarditis: a report of four cases and a systematic review of the literature. J Cardiothorac Surg. 2010;5:13.

\title{
Management of an aortoesophageal fistula caused by Kirschner wire migration in a patient with arteria lusoria
}

\author{
Bastien Orsini, MD, ${ }^{\mathrm{a}}$ Philippe Amabile, MD ${ }^{\mathrm{b}}$ Laurence Bal, MD ${ }^{\mathrm{b}}$ and Philippe Piquet, MD,${ }^{\mathrm{b}}$ Marseille, \\ France
}

Intrathoracic wire migration after orthopedic surgery is a rare complication that most often happens after the treatment of clavicular fractures and dislocation of the sternoclavicular and acromioclavicular joints. We describe here, for the first time to our knowledge, an aortoesophageal fistula caused by intrathoracic Kirschner wire migration.

\section{CLINICAL SUMMARY}

A 58-year-old man treated 6 years previously with a Kirschner wire for a fractured left clavicle was admitted to the hospital for acute thoracic pain. Radiographic examination revealed migration of the wire from the left clavicle to the mediastinum, through the ostium of the left subclavian artery, which was thrombosed, just above the aortic arch. There was no hemomediastinum or pneumomediastinum. We observed that the patient had an arteria lusoria (Figure 1). The tip of the wire was located in contact with the esophagus with an infiltration of mediastinal fat. The patient was in hemodynamically stable condition. Esophageal and bronchial endoscopies were planned, but the patient, who was deaf and nonverbal with mental retardation, refused these examinations.

Open repair was attempted through a median sternotomy. Wire extraction was not possible with this incision alone. A left supraclavicular cervical incision and an anterior thoracotomy in the first left intercostal space were then necessary to control the left subclavian artery and to find the

\footnotetext{
From the Thoracic Surgery Department, ${ }^{\text {a }}$ Hôpital Nord, Marseille, France; and the Vascular Surgery Department, ${ }^{\text {b }}$ Hôpital de la Timone, Marseille, France. Disclosures: Authors have nothing to disclose with regard to commercial support. Received for publication Feb 2, 2012; revisions received April 10, 2012; accepted for publication May 9, 2012; available ahead of print June 7, 2012.

Address for reprints: Philippe Amabile, MD, Aix-Marseille Univ, 13284 Marseille, France and APHM, Department of Vascular Surgery, Hôpital de la Timone, 13385 Marseille, France (E-mail: philippe.amabile@ap-hm.fr)

J Thorac Cardiovasc Surg 2012;144:e25-7

$0022-5223 / \$ 36.00$

Copyright (c) 2012 by The American Association for Thoracic Surgery

http://dx.doi.org/10.1016/j.jtcvs.2012.05.022
}

proximal end of the wire. During extraction, there was no bleeding observed in the surgical site, but blood quickly appeared in the gastric tube, followed by hemorrhagic shock from an aortoesophageal fistula. It was not possible to repair this fistula by sternotomy. A Sengstaken-Blakemore tube was inflated in the esophagus to stop bleeding. The patient was transferred to the intensive care unit for hemodynamic stabilization and then transferred 24 hours later to our hospital, where he was immediately treated.

We decided that an endovascular procedure would be the most appropriate, because there were anticipated difficulties in the surgical control of the posterior aortic arch. The right subclavian artery was transposed in the right common carotid artery, and an aortic stent graft (Cook Inc, Bloomington, Ind) was successfully implanted. Because of the lack of symptoms of left subclavian occlusion, revascularization was not performed. No esophageal injury was seen in the endoscope, so no other complementary procedure was conducted. Broad-spectrum antibiotic treatment was prescribed for 6 weeks.

During postoperative recovery, the patient had difficulties with respiratory weaning that required a tracheotomy and a left pleural decortication. Oral feeding was started without difficulty. Computed tomographic scan showed permeability of the transposition, thrombosis of the lusoria (Figure 2), and absence of mediastinitis. After 1 year of follow-up, the patient is doing well and has regained his preoperative physical state.

\section{DISCUSSION}

Intrathoracic wire migration after orthopedic surgery is a rare complication but has regularly been reported since the first description written by Mazet. ${ }^{1}$ This most often happens after the treatment of clavicular fractures and dislocation of the sternoclavicular and acromioclavicular joints. Many articles have examine the state of tracheal, cardiac, vascular, and esophageal injuries, ${ }^{2-4}$ but to our knowledge 


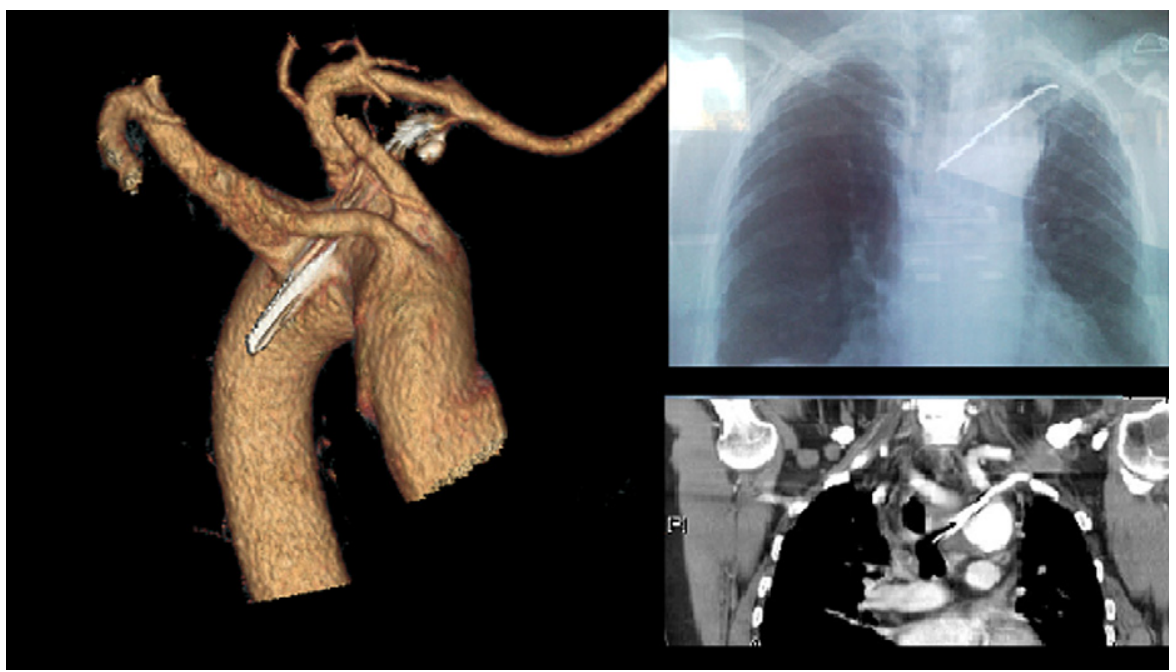

FIGURE 1. Preoperative computed tomographic scan and chest radiograph showing Kirschner wire migration and arteria lusoria.

this is the first time that an aortoesophageal fistula has been described. The existence of an arteria lusoria is a supplementary element involved in this observation that complicated the treatment.

Our observations have brought us to the following conclusions. In case of doubt of aortic injury, one must assume that it is present, and it is not advisable to rely on spontaneous hemostasis, even if the injury is punctiform. Schwartz and colleagues ${ }^{5}$ hypothesized that fibrin is located around metallic wire as the result of an inflammatory reaction..$^{5}$ This reaction is not sufficient to block bleeding once the wire is extracted, as this case demonstrates.

Preoperative evaluation must be exhaustive. An esophageal endoscopy should have been conducted before surgery. If the results had been normal, it would not have been possible to eliminate the possibility of a fistula diagnosis; if the results had been abnormal, however, this would have allowed better planning for the operation and anticipation of possible complications in the postoperative period.
This case illustrates the problem of complex wire removal. There are 2 goals for surgical treatment, to remove the wire to avoid complications and to treat injuries caused by migration. Sternotomy and thoracotomy are commonly used approaches to remove these intrathoracic wires. In our case, a purely surgical intervention aiming to remove the wire (extraction of the bent extremity located under the left subclavian artery) and to repair the aorta and esophagus would have been very complex and invasive, with debilitating consequences for the patient. Endovascular treatment provided a hybrid solution with both endovascular and surgical techniques. In our opinion, this type of intervention should be proposed initially in the treatment of complex migrations involving the aorta in a reference hospital capable of anticipating all phases of this intervention and all complications associated with thoracic surgery.

\section{References}

1. Mazet R. Migration of a Kirschner wire from the shoulder region into the lung: report of two cases. J Bone Joint Surg Am. 1943;25:477-83.

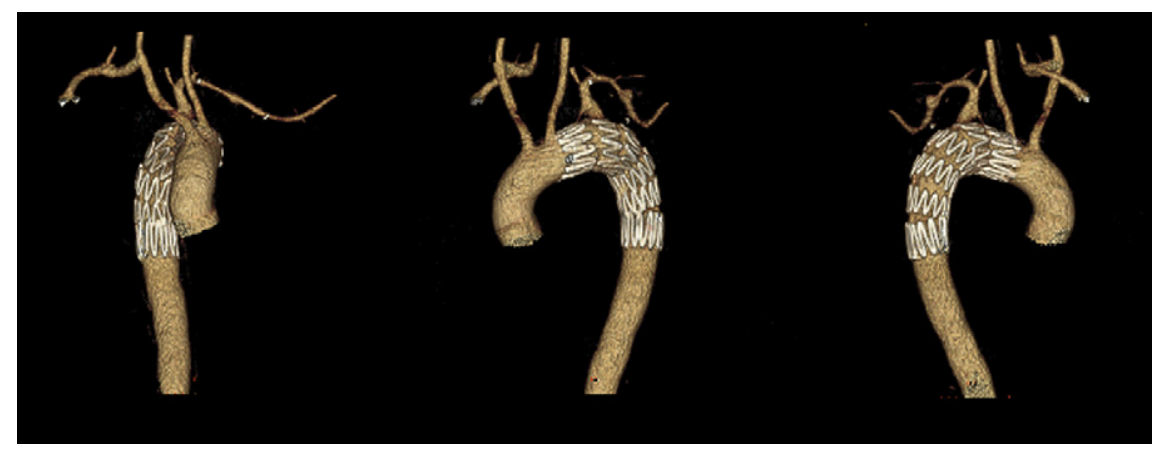

FIGURE 2. Postoperative 3-dimensional computed tomographic scan reconstructions. 
2. Wada S, Noguchi T, Hashimoto T, Uchida Y, Kawahara K. Successful treatment of a patient with penetrating injury of the esophagus and brachiocephalic artery due to migration of Kirschner wires. Ann Thorac Cardiovasc Surg. 2005;11:313-5.

3. Venissac N, Alifano M, Dahan M, Mouroux J. Intrathoracic migration of Kirschner pins. Ann Thorac Surg. 2000;69:1953-5.
4. Nakayama M, Gika M, Fukuda H, Yamahata T, Aoki K, Shiba S, et al. Migration of a Kirschner wire from the clavicle into the intrathoracic trachea. Ann Thorac Surg. 2009;88:653-4.

5. Schwartz A, Thumerel M, Delcambre F, Jougon J. Transaortic migration of a Steinman wire from the shoulder. Eur J Cardiothorac Surg. 2011;40:517-9.

\section{Amplatzer device migration through the sternum: A rare complication of percutaneous treatment for an aortic pseudoaneurysm solved by 2 length-adjustable bovine pericardium conduits}

Ignacio Bibiloni Lage, MD, Stefano Benussi, MD, Alessandro Verzini, MD, and Ottavio Alfieri, MD, Milan, Italy

Pseudoaneurysm of the thoracic aorta after surgery is rare and life-threatening and involves hazardous surgery. ${ }^{1-4}$ Percutaneous strategies may reduce this risk but longterm results are limited. ${ }^{2,3}$ We report a replacement of an aortic graft using 2 bovine pericardial conduits after a failed percutaneous sealing of a perianastomotic thoracic aneurysm resulting from migration of an Amplatzer device (AGA Medical Corporation, Plymouth, Minn).

\section{CLINICAL SUMMARY}

A 43-year-old woman with Takayasu arteritis was admitted with new-onset chest pain 4 months after she had undergone percutaneous closure of a perianastomotic peudoaneurysm. During this period she had remained asymptomatic.

At the age of 30 years she had replacement of the ascending aorta for aortic aneurysm.

Four months earlier, during the study of a 7-year-old cutaneous fistula at the sternotomy wound, 3-dimensional computed tomography (CT) scan revealed a pseudoaneurysm at the distal graft anastomosis $(20 \times 15 \mathrm{~mm}$ with an 8-mm neck), unrelated to the fistula, which had eroded the sternum. Because the operative risk was considered high and cultures were negative without any signs of active infection, the pseudoaneurysm was sealed with a $10-\mathrm{mm}$

\footnotetext{
From the Division of Cardiac Surgery, San Raffaele University Hospital, Milan, Italy. Disclosures: Authors have nothing to disclose with regard to commercial support.

Received for publication Feb 3, 2012; revisions received April 16, 2012; accepted for publication May 15, 2012; available ahead of print June 14, 2012.

Address for reprints: Ignacio Bibiloni Lage, MD, Division of Cardiac Surgery,

San Raffaele University Hospital, Via Olgettina 60, 20132, Milan, Italy

(E-mail: igbibiloni@gmail.com).

J Thorac Cardiovasc Surg 2012;144:e27-8

$0022-5223 / \$ 36.00$

Copyright (C) 2012 by The American Association for Thoracic Surgery

http://dx.doi.org/10.1016/j.jtcvs.2012.05.033
}

Amplatzer muscular ventricular septal defect device. A proximal smaller pseudoaneurysm was left untreated (Figure 1, A).

When she was readmitted, still without signs of infection, CT showed the device in the pseudoaneurysm apex, like an arrowhead, perforating part of its wall (Figure 1, B). Echocardiography revealed mild aortic insufficiency. A surgical approach was therefore imperative.

The right axillary artery and the femoral vein were cannulated. A left ventricular vent was placed through the apex to prevent ventricular distention via submammary minithoracotomy. Cardiopulmonary bypass was established and bladder temperature was lowered to $20^{\circ} \mathrm{C}$. Corticosteroids and thiopental were used for neuroprotection.

During cooling, according to the aortic graft diameter measured by CT, we made 2 bovine pericardium conduits using as a mold a Hegar dilator, each with a double suture of 5-0 polypropylene tied at only one end in order to adjust their lengths afterward (Figure 2, A)

Under circulatory arrest, resternotomy was undertaken resulting in the rupture of both pseudoaneurysms (Figure 2, $B$ and $C$ ). The previous Dacron graft was removed and antegrade cerebral perfusion was administered through the axillary and the left carotid arteries at $800 \mathrm{~mL} / \mathrm{min}$ and $400 \mathrm{~mL} / \mathrm{min}$, respectively, while the innominate and the left subclavian arteries were occluded. The distal aorta was anastomosed to one of the readymade conduits and CBP was resumed after the distal graft had been clamped. The second conduit was sutured to the sinotubular junction and the 2 conduits were anastomosed to each other with 4-0 polypropylene. Cardiopulmonary bypass and circulatory arrest times were 203 minutes and 46 minutes, respectively.

Staphylococcus epidermidis grew on the culture of the graft biopsy. The patient was discharged on postoperative 